\title{
Composite modelling of interactions between beaches and structures
}

\section{Herman Gerritsen ${ }^{1}$, James Sutherland ${ }^{2}$, Rolf Deigaard ${ }^{3}$, Mutlu Sumer $^{4}$, Conceição J.E.M. Fortes ${ }^{5}$, Joan-P. Sierra ${ }^{6}$ and Ulrike Schmidtke}

\footnotetext{
${ }^{1}$ Herman Gerritsen (IAHR Member), Deltares, Rotterdamseweg 185, 2600 MH Delft, the Netherlands. herman.gerritsen@deltares.nl (author for correspondence)

2 James Sutherland (IAHR Member), HR Wallingford, Wallingford, Oxfordshire OX10 8BA, UK. j.sutherland@hrwallingford.co.uk

${ }^{3}$ Rolf Deigaard, DHI, Agern Allé 5, DK-2970 Hørsholm, Denmark. rd@dhigroup.com

${ }^{4}$ Mutlu Sumer, Technical University of Denmark, Nils Koppels Allé, DK-2800 Kgs. Lyngby, Denmark. bms@mek.dtu.dk

${ }^{5}$ Conceição J.E.M. Fortes, National Laboratory for Civil Engineering. Av. do Brasil, 101. 1700-066, Lisbon, Portugal. jfortes@Inec.pt

${ }^{6}$ Joan-P. Sierra, Laboratori d'Enginyeria Maritima, Universitat Politecnica de Catalunya, Jordi Girona 1-3, 08034 Barcelona, Spain.

joan.pau.sierra@upc.edu

${ }^{7}$ Ulrike Schmidtke, Coastal Research Center, Leibniz University Hannover and Technical University Braunschweig, Merkurstrasse 11, 30419 Hannover Germany.schmidtke@fzk-nth.de
}

Published in the Journal of Hydraulic Research. 49:sup1, 2-14

\section{Abstract}

An overview of Composite Modelling is presented, as elaborated in the EU/Hydralab joint research project Composite Modelling of the Interactions Between Beaches and Structures. An introduction and a review of the main literature on composite modelling in the hydraulic community are given. In Section 3 , the case studies of composite modelling of the seven partners participating in this project are discussed. The focus is on the methodologies used and their impact on the modelling approach, rather than the results of the experiments per se. A further section presents reflections on key elements in composite modelling, as they emerged in the various case studies. The related subject of Good Modelling Practice is summarized in Section 5. Then guidelines are given on how to decide if composite modelling may be beneficial, and how to set up a composite modelling experiment. It is concluded that composite modelling in the hydraulic community is still in its infancy but involves challenging research with significant potential.

\section{Keywords}

Beach, composite modelling, hydraulic scale modelling, numerical modelling, structure

\section{Introduction}

This research presents a concise introduction to and overview of the 'Guidelines for Composite Modelling of the Interactions Between Beaches and Structures (CoMIBBS)' (Gerritsen et al. 2009, Gerritsen and Sutherland 2011). These Guidelines constitute the final product of the EU/HYDRALAB-III Joint Research Activity of CoMIBBS. The authors led their institute's contribution to the composite modelling in CoMIBBS. The Guidelines describe techniques for combining physical and numerical modelling to improve the physical modelling infrastructure. Composite modelling can lead to different forms of improvements to model problems that can not be tackled by either physical or numerical modelling alone, increasing quality at the same cost or obtaining the same quality at reduced cost, and / or reducing uncertainty at the same cost, 
since uncertainty reduction is also a quality issue. The purpose of the guidelines is to assist modellers in the process of making decisions on how to conduct modelling and they are expected also to serve as inspiration for the academic community. Although not specifically treated herein, composite modelling of other nearshore processes may benefit from the methodologies and techniques described herein. Since composite modelling in the field of coastal engineering is a rather new approach, and relatively little was published so far, the focus is on key literature references and on summaries of the eight composite modelling study cases that were conducted by the seven research teams in the HYDRALAB CoMIBBS project.

\section{Concept of composite modelling}

The links between the four building blocks of our knowledge on (hydraulic) processes in coastal engineering and behaviour can be visualised by Fig. 1. Each element encapsulates a part of our hydraulic knowledge, theory and application experience and so makes this objective, transferable and verifiable. Combining physical and numerical modelling, or composite modelling, may open up new opportunities for physical modelling, e.g. lead to a new generation of physical modelling infrastructure. Its definition here is: "Composite Modelling is the integrated and balanced use of physical and numerical models".

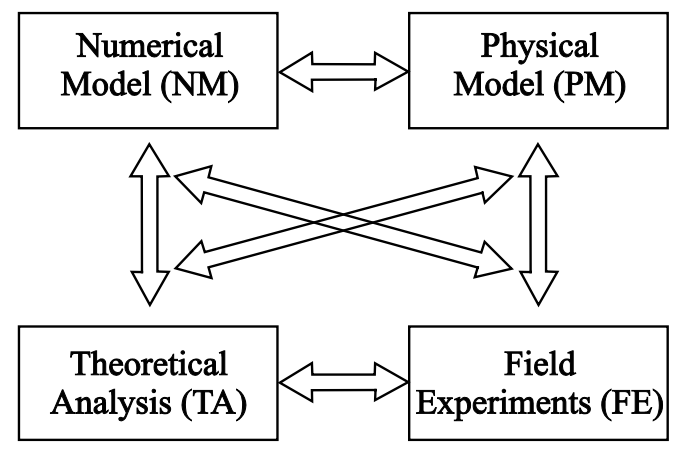

Figure 1: Links between four elements of research methodology in coastal engineering knowledge

Several ideas and expectations are associated with Composite Modelling (CM). Firstly, CM allows to obtain the best out of both Physical Models (PM) and Numerical Models (NM) for a given problem by applying each for that geographical area or for those scales where it performs best, and so lead to an overall optimum simulation of the relevant processes. Secondly, it may provide more quality (higher accuracy, reduced uncertainty) against the same cost and, thirdly, it may provide the capabilities to model more complex problems which individual PM or NM cannot.

Composite modelling was discussed in the past, though often with slightly different definitions. An example is Barthel and Funke (1989), who referred to as 'hybrid modelling'. Oumeraci (1999) proposed a move towards composite modelling by combining the main strengths of physical and numerical models within a formal framework for exchanging information. As part of a composite model, physical model tests would be conducted at a large enough scale to minimise scale effects, concentrating on important processes that are not well enough understood to be modelled accurately in a numerical model, resulting in interactions of short-scale and long-scale processes. The results are analysed to increase process understanding of the system behaviour but are also parameterised so that they can be incorporated into a numerical model to cover a wider area. Kamphuis (2000) discussed the use of various types of modelling and the need to provide well-based quantified results in coastal engineering. He advocated an integration of prototype data, physical and numerical modelling as a further element to improve modelling and design. These elements can 
essentially complement each other, because model simulations provide the continuum information to bridge the gaps in field data. A key element in integrated models is the computational module "information manager" (Fig. 2).

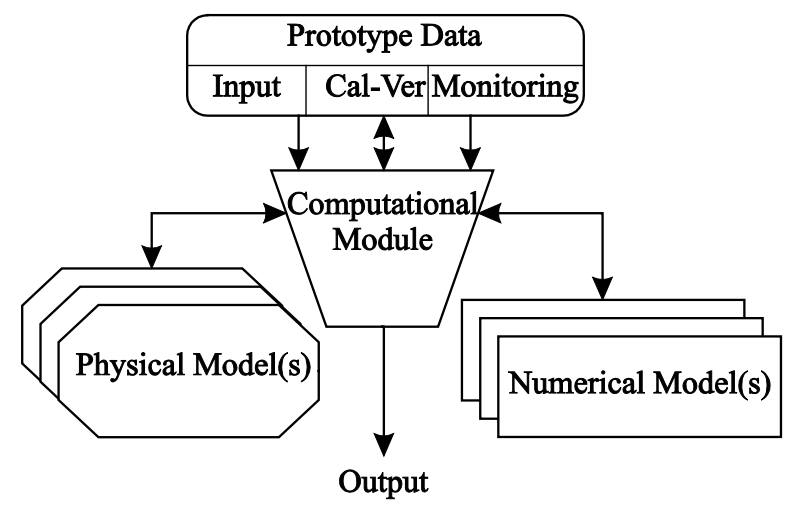

Figure 2: Model integration leading to an integrated model

van Os et al. (2004) combined the elements of Fig. 1, applying each for those aspects or problem parts where they are most suited. The authors argue that this integration of numerical modelling systems, field measurements, experimental laboratory research and theoretical analysis, combined through 'calculation or integration modules' will lead to what is called 'hybrid modelling' or 'composite modelling'. For a problem with different spatial scales, modelling the far field by numerical modelling, and the near-field with physical modelling, with a proper exchange of information between the two, information exchange and results can be tied together computationally. The authors list a range of topics that have been identified and are suited for $\mathrm{CM}$ in hydraulic modelling.

\section{Composite Modelling techniques used by COMIBBS}

\subsection{Case studies}

Seven organizations carried out case studies during the CoMIBBS project, namely:

- 3.1: Coastal area NM provided boundary conditions to local wave basin PM;

- 3.1: Coastal area NM was used to design a wave basin PM;

- 3.1: Wave basin PM provided boundary condition within regional 1-line NM;

- 3.2: Phase resolving wave NM was used to reduce effects of bathymetry uncertainty in wave flume PM.

- 3.3: Wave flume PM was used to calibrate a phase-resolving wave NM, then to quantify the errors in PM and NM at different scales;

- 3.4: Wave basin PM was used to undertake a sensitivity analysis of a coastal area NM;

- 3.5: Parameterization of processes from PM passed to RANS NM and validated against a different current flume PM;

- 3.6: Fitted parameters in a scour formula were re-calibrated using large-scale PM results (as NM and PM results were different); 
- 3.7: Results from coastal area NM and wave flume PM were combined using a priori knowledge of strengths and weaknesses;

Each of the seven case studies is described below. Extended descriptions of the case studies are given by Gerritsen et al. (2009) or Gerritsen and Sutherland (2011).

\subsection{Sedimentation bypass for a harbour layout}

Grunnet et al. (2008ab) considered a harbour placed on an exposed straight coastline and investigated the conditions for maximum bypass of sediment and minimum sedimentation in the harbour mouth by applying composite modelling, involving:

1. Nesting of a local model within an area model;

2. Use of a coastal area NM to design a local PM; and

3. Use of PM results to influence a regional 1-line model of beach plan-shape evolution.

Each of these methods is described below.

A regional numerical wave model for a straight coastline was used to transform offshore wave conditions to the boundary of a nested hypothetical physical model of a harbour protected by breakwaters. The regional model used for nesting should include the most important physical processes needed to transform offshore conditions to inshore. In this case these were wave propagation, depth refraction and energy dissipation. A coastal area numerical model was then used for the design of an idealised PM including the size of the harbour in the PM, the harbour location between the lateral sides of the basin and the seaward distance to the wave maker to ensure that the blocking effect did not cause problems in the PM. In addition, numerical modelling allowed for an initial assessment of the conditions for sediment transport to ensure that sediment would in fact be mobilized in the down-scaled laboratory experiment.

The basin for the PM was one of DHI's shallow water basins $35 \mathrm{~m}$ long, $25 \mathrm{~m}$ wide and $0.80 \mathrm{~m}$ deep. A $17 \mathrm{~m}$ long 3D wave maker was fixed along one side of the basin generating irregular, multidirectional waves. In the hydraulic scale model, the harbour had to be placed far enough from the inflow boundary to obtain a good cross-shore distribution of the fully developed current. To determine this location, a NM including the physical constraints and boundary conditions described above was run against a NM with periodic lateral boundary conditions representing the idealised uniform coast. In both models, the current was extracted along cross-shore profiles defined between $x=0$ and $x=2,500 \mathrm{~m}$. Figure 3 shows current profiles, and indicates that these from the uniform and the prototype NMs fit perfectly at $x=1,000 \mathrm{~m}$. Thus the harbour entrance could be placed at this location. 


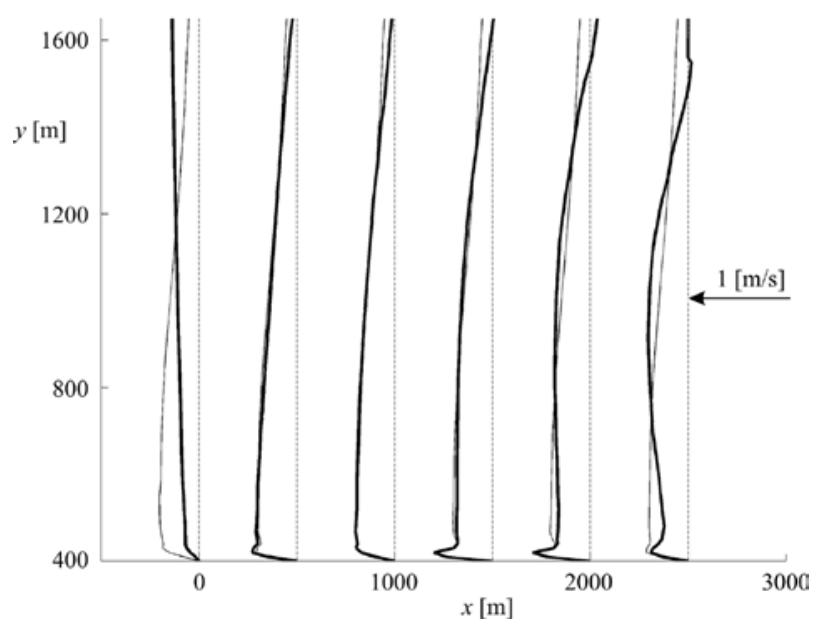

Figure 3: $(-)$ Current velocity distribution extracted along $x$-direction from uniform, periodic model, $(-)$ laboratory model

Due to the dimension of the physical basin, the PM was defined with a length scale of 1:100. According to Froude's model law, the corresponding time scale was 1:10. The geometry of the wave guides was determined in the NM of the physical scale model to ensure wave conditions as close to the uniform conditions as possible. Wave-driven currents from the NM are presented in Fig. 4.

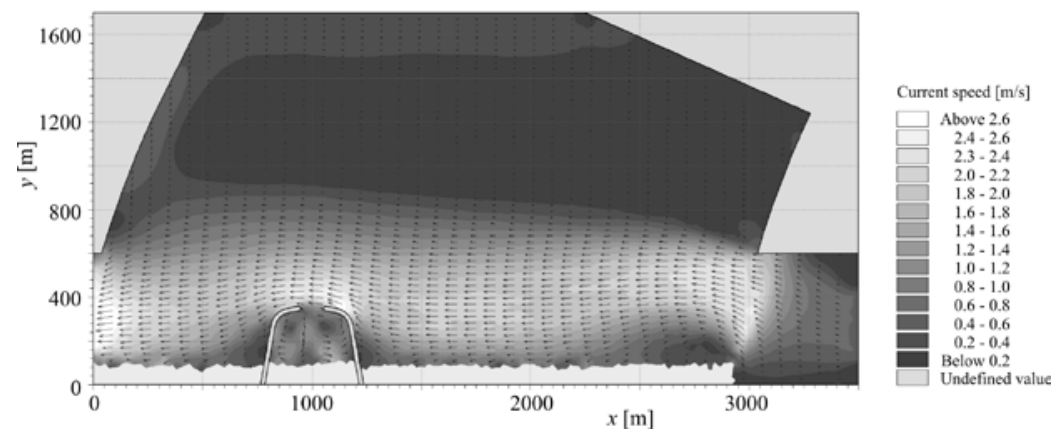

Figure 4: Wave-induced current magnitude and direction for physical model domain simulated by NM; note that wave guides following wave rays at each end of wave generator front are included in model

A comparison between the inflow magnitude in the PM and the NM at $x=3,000 \mathrm{~m}$ was established to ensure that the forcing at the boundary is similar for both models. The results are shown in Fig. 5 . Note that the inflows from the PM and the NM have the same patterns and both tend to increase into deeper water, while the NM tends to give too small velocities at the more shallow inner part of the profile.

The deposition of sediment in the harbour basin was determined in the physical model. Simulations were made with a coastline model to determine the regional coastline evolution caused by sediment blocking by the harbour and sedimentation in the harbour basin. The blocking of the longshore transport and the rate of sediment deposition in the harbour are internal boundary conditions for the regional coastline model, which was specified on the basis of the results and observations from the PM. The conditions for the regional model were thus obtained based on the results from the local PM. Simulations were made for various amounts of sedimentation in the harbour and with different assumptions regarding how the sediment that bypasses the harbour is distributed along the coastline down-drift of the harbour. 


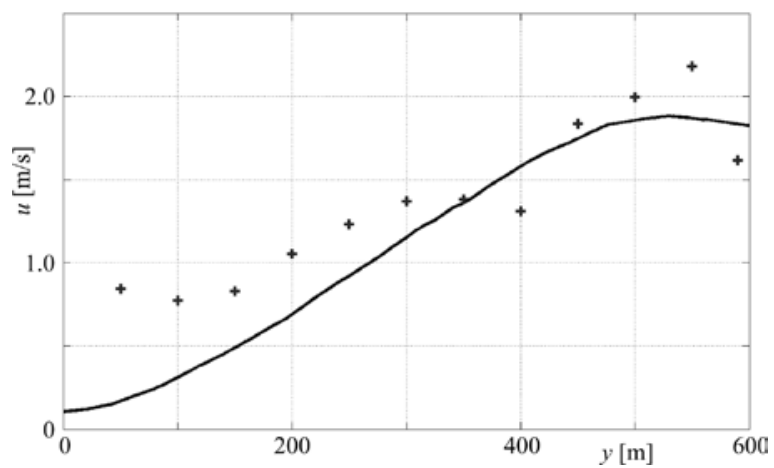

Figure 5: Comparison of inflow current speed at X=3,000 $\mathrm{m}$ between $(-)$ numerical results and $(+)$ physical measurements

Three forms of composite modelling are described in this case study. The model nesting indicated that the numerical models can transfer the forcing conditions from locations far from the PM domain to the boundary of the model or to locations inside the domain. From the second example the authors conclude that the NM was useful to design the set-up of the physical experiments. In particular the NM was able to represent the conditions in the test basin well enough to determine the maximum allowable model scale and to determine the optimal location of the harbour in the model basin. The third form of CM indicated how detailed information obtained from the PM may in turn be transferred to a regional model, where the modelling of coastline evolution was improved on the basis of findings from the PM.

It is recommended that, when conducting physical scale tests, the emphasis should be on optimising the scale and model technique of the physical experiments. The PM can be considered as a local model. Rather than attempting to increase its area by increasing the model scale, $\mathrm{CM}$ should be used to transfer regional data on the forcing to the boundaries of the PM.

\subsection{Reduction of uncertainties in physical modelling}

Sandy near-shore bottom profiles can change significantly under severe storms, and it is therefore desirable to take potential variations in bathymetry into account in PM of the (most critical) wave loads on coastal structures (Fig. 6). For reasons of complexity and cost, the uncertainty in bathymetry is hardly ever taken into account in PM, however.

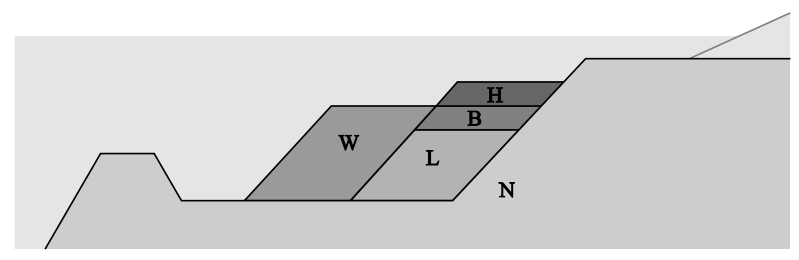

Figure 6: Schematisation of foreshore of Petten Sea defence structure (distorted scale), with from left to right: deep water, bar, trough, five foreshore terrace variants (No, Low, Basic, High and Wide terrace), toe and spending beach

With numerical simulation, this can be done much more flexibly and efficiently, but unfortunately NM results for these processes do not yet have the same accuracy as physical scale experiments. In this case study CM was used to efficiently and accurately determine the bed form that gives the maximum damage at the toe of 
the structure. CM was used to reduce the uncertainty in PM in which merely one or two 'well chosen' bed forms are considered. Previous studies indicated that the damage at a toe due to wave attack is a function of Target $_{1}=H_{s}^{0.5} \cdot T_{m-1,0}$, with $H_{s}$ as the spectral significant wave height and $T_{m-1,0}$ as the spectral mean wave period.

van den Boogaard et al. (2009ab) considered the 1D case of waves over a typical schematised foreshore in front of a seawall. The foreshore has a bar and a Low Tide Terrace (LTT) with unknown length and height $\left(\alpha_{1}, \alpha_{2}\right)$, representing the variation in the foreshore profile (Fig. 6). They have modelled exactly the same problem with a PM (Scheldt Flume) and with a NM (phase resolving Boussinesq model TRITON). This allowed a one-to-one comparison of the results of both approaches in terms of wave height $H_{s}$, wave period $T_{m-1,0}$ and water level $h$ at all measurement locations, for a range of relevant boundary forcing $\left(H_{s}, T_{m-1,0}\right.$ and h). The comparison and data analysis indicate a systematic dependency of the wave conditions at the toe of the structure on the offshore hydraulic conditions and the length and height of the low tide terrace $\left(\alpha_{1}, \alpha_{2}\right)$. The wave conditions at the toe obtained from the PM were taken to be correct. The composite modelling approach consisted of first deriving a dedicated model for the systematic errors in the NM from a limited number of identical simulations with PM and NM. This error correction model was based on a neural network of Multilayer Perceptron type MLP2.5, which was trained on the results of the identical PM and NM simulations for five bed forms $\left(\alpha_{1}, \alpha_{2}\right)$, each for twelve combinations of relevant boundary forcing of $\left(H_{s}, T_{m-1,0}\right.$

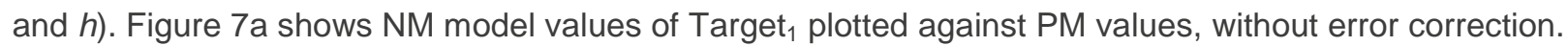
Figure $7 \mathrm{~b}$ shows the same results after the error correction model had been applied to the NM results.
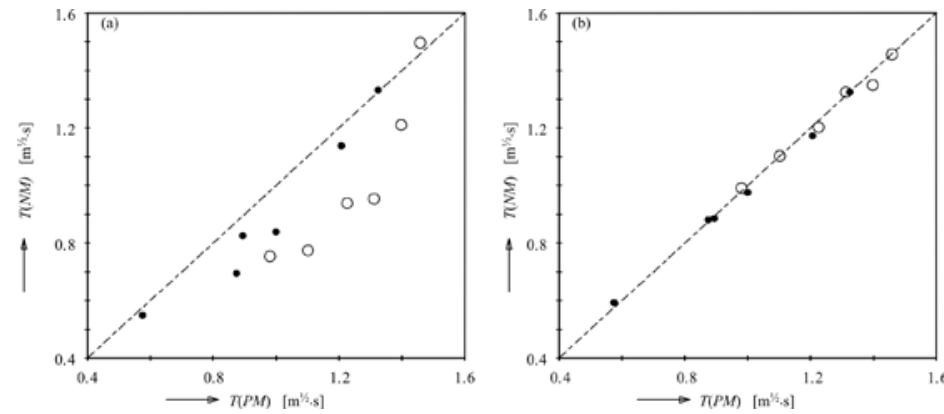

Figure 7: Scatter plots of relative numerical model error (a) before, (b) after application of correction, for $T=$ Target $_{1},(---)$ equal values from NM and PM; $(\bigcirc)$ low, $(\bullet)$ high water cases, respectively

Secondly, the most critical bed form parameters $\left(\alpha_{1}, \alpha_{2}\right)$ were determined via efficient NM sensitivity simulations in which $\left(\alpha_{1}, \alpha_{2}\right)$ were systematically varied, and the corresponding wave damage levels determined. The sensitivity simulations showed little variation with $\alpha_{1}$, so the focus was on variations in LTT height $\alpha_{2}$. The third step was the application of the error correction model to the NM results. This led to corrected NM results of wave damage as function of $\left(\alpha_{1}, \alpha_{2}\right)$ that have accuracy of near -physical model result quality. The bed form parameters for which wave damage is a maximum are the most critical ones (Fig. 8). The maximum wave damage was found for $\alpha_{2}=0.15$, if $\alpha_{1}=0.163$.

The thorough prior analysis of the nature of the differences between NM and PM results guided the construction of a successful (neural network-based) error correction procedure. After application to NM results, identification of, and conclusions on most critical situations were made much more accurately and reliably than on NM results as such. As a whole, this methodology significantly reduced the number of flume experiments to derive such critical conditions by PM only. 


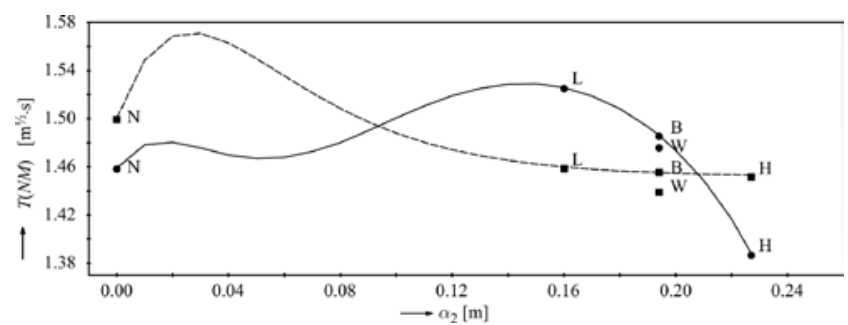

Figure 8: Optimisation of wave-load versus height $\alpha_{2}(--)$ before, $(-)$ after error correction for the five foreshore terrace variants

\subsection{Determination of optimum physical model scale}

The accuracy of modelling wave breaking and sediment transport near the vicinity of coastal structures using laboratory experimentation depends much on the model scale used. A priori, the optimum scale is not known. This case study derived methods of determining relationships between model scale and errors.

Fortes et al. (2009), Reis et al. (2008) and Lemos et al. (2009) studied the effect of the PM scale and the representation of processes in a NM on (i) the simulation of wave propagation on coastal defences, in particular where the wave breaking phenomenon plays a role, and (ii) the beach profile evolution in front of alongshore structures such as seawalls/revetments (Freire et al. 2009). For wave breaking they used a multilayer, nonlinear, phase-resolving NM (COULWAVE, Lynett and Liu 2004) to define the smallest scale to be used in PM tests in a flume that complies with a pre-set level for model scale effects of the wave propagation on a plane slope that ends on a coastal defence. Conversely, scale model results were used to fine-tune the uncertain numerical model parameters to better describe the flow, especially the wave breaking position and height. For the sediment dynamics, experiments of the beach profile evolution under storm and mild wave conditions were conducted at two laboratory scales. These were combined with numerical modelling (LITPROF, DHI 2007), to assisted the design of experiments, and to evaluate its strengths and weaknesses with respect to scaling problems.

The first problem focused especially on the complex physical processes involved in the breaking zone. Tests were undertaken using 1:10, 1:20, 1:30, 1:40 and 1:60 model scales, considering regular and irregular incident wave conditions. Some tests were used to calibrate the model. The validation of the composite modelling methodology consisted of comparing the scale effect error for wave breaking height and position obtained with the NM for two selected test cases not used in the calibration of the numerical model. The scale effect errors are stated in Table 1.

Table 1: Physical and numerical errors in wave breaking position $L_{b}$ and wave breaking height $H_{b}$ due to scale effects

\begin{tabular}{|l|l|l|l|l|}
\hline \multirow{2}{*}{ Scale } & \multicolumn{2}{|l|}{$L_{b}$} & \multicolumn{2}{l|}{$H_{b}$} \\
\cline { 2 - 5 } & Error_PM & Error_NM & Error_PM & Error_NM \\
\hline $1: 20$ & $2.9 \%$ & $2.9 \%$ & $6.1 \%$ & $0.1 \%$ \\
$1: 40$ & $2.9 \%$ & $2.9 \%$ & $18.2 \%$ & $1.2 \%$ \\
$1: 60$ & $2.9 \%$ & $0.1 \%$ & $27.1 \%$ & $3.9 \%$ \\
\hline
\end{tabular}


For the second problem (Freire et al. 2009), the composite modelling approach shown in Fig. 9 was taken to find the best small-scale PM to simulate the beach profile evolution in a realistic way. Two different scales were tested, namely a large-scale (1:6) and a small-scale (1:13) model.

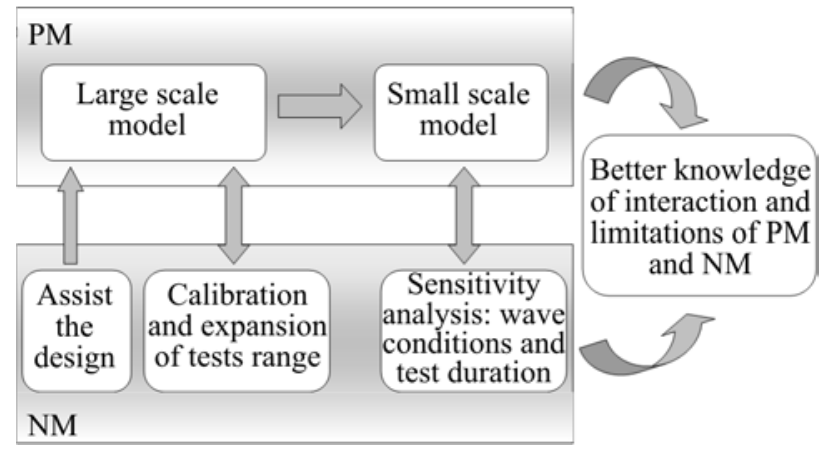

Figure 9: Schematic view of composite modelling approach for modelling sediment dynamics

PM model tests were performed for erosive and accretive conditions in a large and a small-scale wave flume. The NM was applied to simulate the large-scale tests, for calibration and validation. Based on the numerical results, modifications of the initially planned wave input conditions were tested in the PM. The sensitivity analysis performed with the NM, and the lack of agreement between the small-scale NM and PM results confirmed that the morphological behaviour of the beach profile at the small-scale is not a scaled-down version of the corresponding behaviour at the large-scale.

Key findings for wave modelling are that the NM helped in the design of the PM tests and in the set-up of the test equipment. Further, the NM was able to predict the physical model errors due to scale effects associated with the wave breaking position, but not these associated with wave breaking height. The composite modelling of the morphological behaviour of the beach profile evolution was found to greatly improve the results and conclusions that would had been possible to gather if only one technique had been used. Moreover, CM allowed the optimization of the use of PM.

Generally, the authors conclude that $\mathrm{CM}$ is an adequate tool to study wave propagation since it contributes to significant improvement of the model results compared to either PM or NM. For sediment modelling, they conclude that $\mathrm{CM}$ to study the beach profile evolution performed can be generalised.

\subsection{Sensitivity analysis using skill scores}

Waves and sedimentation around detached breakwaters have been modelled using PM and NM, which both have their strengths and weaknesses (Sutherland and Obhrai 2009) including:

- PM strengths: nonlinear processes; local scour and diffraction;

- PM weaknesses: scale effects;

- NM strengths: can model a set of breakwaters without scale effects;

- NM weaknesses: simplified processes, especially scour, diffraction and representation of breakwater with no swash-zone processes.

The PM of detached breakwaters was made in a coastal wave basin with a single detached offshore breakwater in the centre of a bed of fine sand of $d_{50}=0.11 \mathrm{~mm}$ (Obhrai and Sutherland 2009). Five tests were run, only with changes to the representation of the water levels, currents and transmission of the 
breakwater. A laser scanner was used to measure the full physical model bathymetry, which was then transferred to the NM. An example final model bathymetry is shown at full scale in Fig. 10a.

Numerical modelling was undertaken using the coastal area model PISCES, which used the finite element flow model TELEMAC, the third generation coastal area wave model SWAN and the sand transport module SANDFLOW to simulate sediment transport, which drove changes in the bathymetry. NM simulations were performed of full-scale versions of the PM, with variations in model set-up. An example final NM model bathymetry is shown in Fig. 10b.

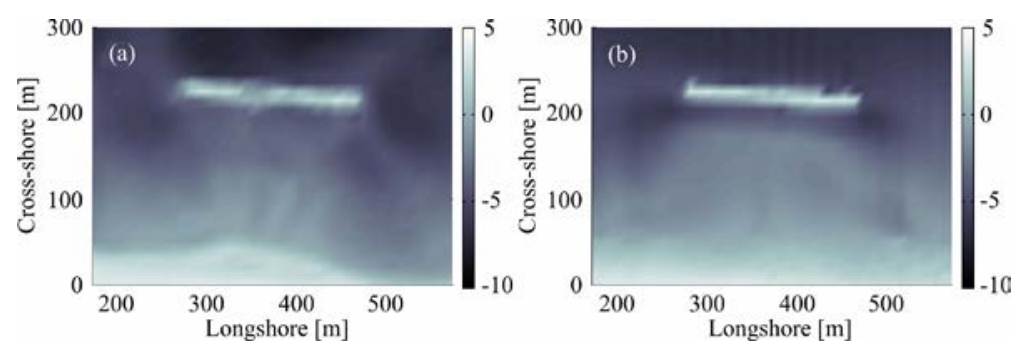

Figure 10: (a) Full-scale final bathymetries from (a) physical model, (b) numerical model

The CM hypothesis tested was that a quantitative technique for optimising the information flow between models could be developed using skill scores (Sutherland et al. 2004). The optimum information flow is determined by calculating the skill of each model run. The skill scores gives a quantitative measure of the incremental benefits of adding additional processes or using more complicated boundary conditions (Sutherland and Obhrai 2009). This would allow for an optimal model setting to be chosen resulting in a high skill score without using excessive resources.

For this objective, a series of NM and PM model runs were conducted to test the sensitivity to variations in the set-ups of both models. Neither provides a set of correct results everywhere, as both have weaknesses. Therefore the choice was made to perform a comparative analysis on part of the model domain, in this case an area inshore from the breakwater, where the PM was judged to give the best results and was taken as the correct bathymetry in the Brier Skill Score. An example set of results from the numerical model of Test 1 is given in Table 2. The Brier Skill Scores in Table 2 state that including diffraction in the NM was more important than including transmission. The skill scores are all relatively high indicating that the NM was capable of generating a recirculation current that produced accretion and the formation of a salient, similar to that in the PM. Further information on the type of errors involved comes from studying the components of the Brier Skill Score $B S S=(J-K-L+M) /(1+M)$. The position accuracy $J$ was broadly similar for the 4 NM runs of Test Series 1, indicating that the patterns of deposition and erosion were similar. The transport rate error term $K$ which should be as low as possible, was much lower for cases with diffraction than without. In all cases there was a significant normalisation term $M$ indicating that there was a significant change in the measured average bed level, caused by the net import of sediment into the area considered. The sediment budget error $L$ was much lower than the normalisation term $M$ showing that the NM imported approximately the same volume of sand as the PM. Various further PM and NM experiments were performed with different settings that required different levels of computer, laboratory and human resources to run and which required different information to be passed between models. The skill of each run in simulating the evolution of the salient was calculated, which allowed an optimum model set-up to be chosen that had a high skill score but did not require excessive resources to run. 
Table 2: Example skill scores from numerical model of Test 1

\begin{tabular}{|c|c|r|r|r|r|r|}
\hline Diffraction & Transmission & BSS & J & K & L & M \\
\hline Yes & Yes & 0.58 & 0.34 & 0.02 & 0.02 & 0.67 \\
\hline Yes & No & 0.58 & 0.32 & 0.02 & 0.001 & 0.67 \\
\hline No & Yes & 0.45 & 0.28 & 0.13 & 0.07 & 0.67 \\
\hline No & No & 0.42 & 0.27 & 0.15 & 0.09 & 0.67 \\
\hline
\end{tabular}

This procedure forms an objective method for undertaking a sensitivity analysis using the BSS. Additional information on the error sources was obtained using the components of the BSS. Using a quantitative measure of skill to assess model sensitivity represents a move away from the use of subjective judgement in choosing a model set-up. However, the objectivity of the method was compromised because there was no truly correct final bathymetry: the skill score with PM and NM bathymetric change is a measure of the accuracy of a prediction (compared to the correct outcome) relative to the accuracy of a baseline prediction (compared to the correct outcome) in situations where there is no model that predicts the correct outcome. Here a subjective analysis of the strengths and weaknesses of the PM and NM was used to select an area where there was a best model, which was used as the 'correct' model in the skill score. Hence, relative skill scores were calculated.

\subsection{Composite modelling of scour with parameterised turbulence from a physical model}

The flow around a solid half-buried sphere in a sandy seabed can cause additional turbulence, compared to the case without the solid body. The understanding of the effect of this externally generated turbulence on scour cannot be achieved by applying only a PM or a NM (Sumer 2007). A CM approach was therefore designed to systematically investigate the processes involving the externally generated turbulence and its role on scouring. This approach allows the externally generated turbulence in the model runs to be switched on and off to single out its effects, and to shed light onto these complex processes.

The key of the CM is the transfer of turbulence determined in the PM to the NM. The NM consists of two numerical codes: (a) hydrodynamic code Ellipsys3D (a 3D RANS solver). Turbulence viscosity is calculated by the $k-\Omega$ shear-stress transport closure model in the present application and (b) morphologic code comprising three components for describing the: (1) sediment transport process (Engelund-Fredsøe bedload equation in vectorial form), (2) sand slide process (bed avalanches when the slope exceeds the angle of repose), and (3) mass balance for sediment (at each grid point on bed, to solve bed elevation $h$ ). A half buried spherical object was placed in the PM to represent an element of a scour protection layer (Dixen 2008, Dixen et al. 2011). 

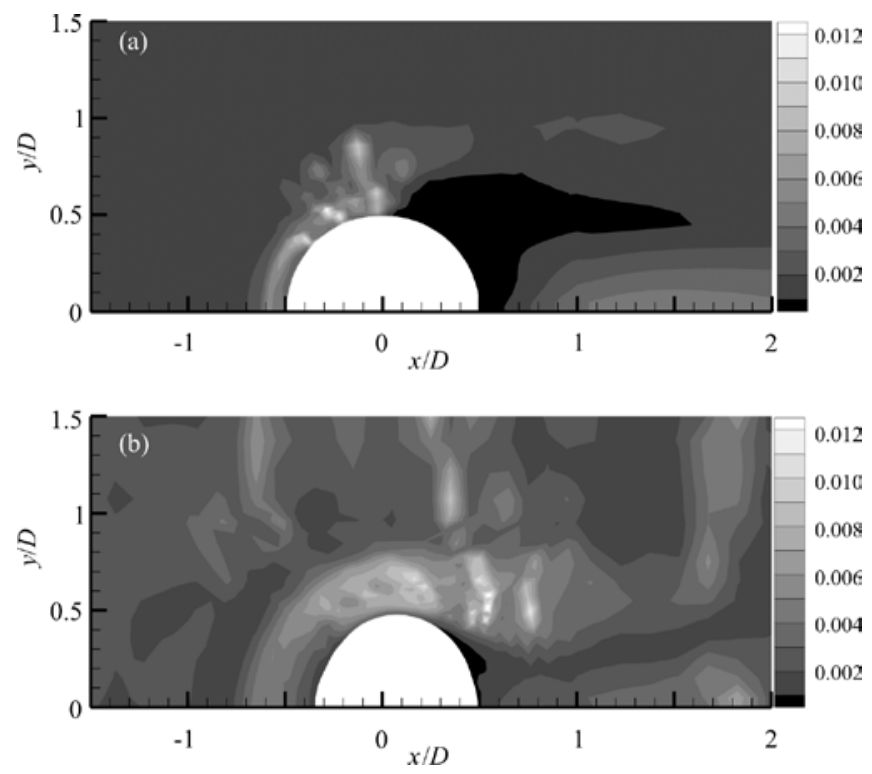

Figure 11: NM determined turbulent kinetic energy $k$ for (a) initial stage of scour; plane bed, (b) equilibrium stage; scoured bed

The flow around a bottom-seated hemisphere has two key features: a horseshoe vortex in front of the sphere, and lee wake behind it, with shed, arch vortices. The flow underneath these areas exhibits strong turbulence (Fig. 11), where $y=$ elevation above seabed, $x=$ downstream distance and $D=$ sphere diameter. Figure 11 shows the Turbulent Kinetic Energy (TKE) $k$ for two stages of the scour process, calculated from the present NM, indicating that the sphere introduces an additional field of turbulence. The implication of the latter is that the sediment transport and therefore the scour differs from that described by the classic sediment transport formulae.

The morphological model was modified to account for the influence of the additional turbulence. This is essentially what was done in the present CM study. The modelling procedure is as follows:

1. Run the numerical model for a half-buried sphere;

2. From the numerical model results, find the ranges of $k$ during the development of the scour hole;

3. Conduct physical model experiments. In the experiments, adjust the turbulence generation so that the range of external field of turbulence is the same as that identified in NM;

4. From the PM, find the sediment transport versus the externally generated turbulence, i.e. $q_{b}=f(k)$;

5. Feed Eq. (1) into the NM, and run it to calculate the scour.

The results of the present $\mathrm{CM}$ exercise show that while the equilibrium scour depth is not influenced by the effect of externally generated turbulence, the time scale of scour is affected markedly. The equilibrium scour depth is uninfluenced because the plan-view extents of the horseshoe and lee-wake vortices are the same regardless of whether or not the externally generated turbulence in the calculations is On or Off. The equilibrium scour depth is about $S / D=0.45$. The time scale of scour with the externally generated turbulence On is shown to be by a factor 2 smaller than that with the turbulence Off. This is not unexpected because the sediment transport occurs faster due to the presence of 'additional' turbulence. This transfer of information from the PM to the NM allowed for the scour calculation in the NM to be improved substantially. The latter 
enables to run both PMs and NMs at the same time to obtain a more accurate picture of the scour process, which was not possible before the present CM exercise.

\subsection{Parameterisation of physical model results for composite modelling}

The topics of interest of this case study are the processes around a vertical slender monopile in waves and the improvement of knowledge about the dimensions of the developing scour in non-cohesive sediment, such as modelled in Hannover's large-scale GWK experimental facilities. Combining the best fitting numerical model for scour development and the results of the physical model through a smart CM approach was thought to give further insight. Therefore, a review was made of all general existing tools for CM: numerical modelling, physical modelling, analytical modelling and field measurements (Prepernau et al. 2008), indicating that data from field measurements are still not available. It also became clear that no suitable NM is as yet available for simulating wave-induced scour around monopiles. The final bathymetry from a PM test of scour due to irregular waves is presented with the final bathymetry from a NM simulation of the same experiment (Göthel 2008) in Fig. 12. The NM underestimated the scour depth and showed a different pattern of scour and accretion, due to scale effects or differences in the representation of processes, such as undertow and wave asymmetry between the NM and the PM.

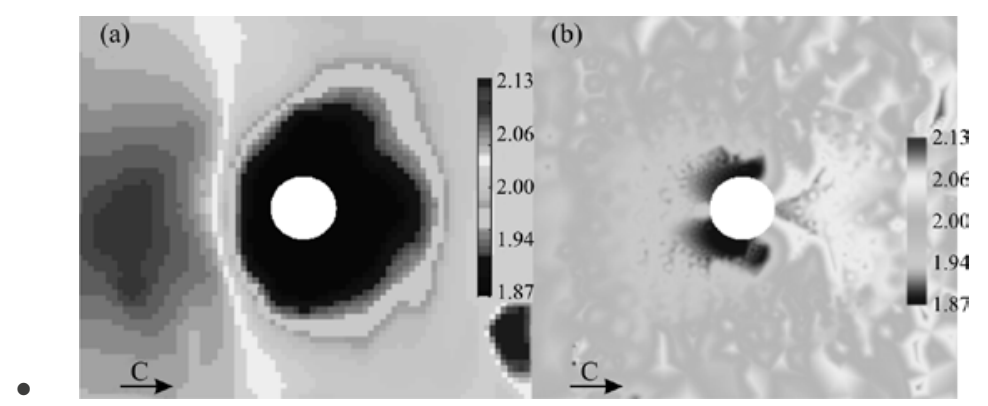

Figure 12: Scour depth from (a) large-scale PM model test, (b) state-of-the-art NM; C denotes current direction

As an alternative to a CM in the sense of Oumeraci $(1999,2009)$, the fitted parameters in the scour formula of Sumer and Fredsøe (2001), which were derived from small-scale PM tests possibly affected by scale effects, were recalibrated using the results from the large-scale PM laboratory tests. The scour formula is

$$
\frac{S_{c w}}{D}=\frac{S_{c}}{D}[1-\exp (\{-A(K C-B)\}]
$$

where $S_{c}$ and $S_{c w}$ denote scour depth induced by current and current plus waves, $D$ is the pile diameter, $A$ and $B$ are fit parameters and $K C=u T / D$ is the Keulegan-Carpenter number, with $u$ and $T$ as velocity and period. This model predicts the equilibrium scour depth induced by combined waves and steady currents. For wave spectra Sumer and Fredsøe (2001) recommended the use of the root-mean-square velocity $u_{\text {rms }}$ and the peak wave period $T_{p}$.

Case 1 tests were run with an initial even bottom at test start. It was found that the formula improves if $K C$ is based on the maximum wave velocity $u_{\max }$ and the mean wave period $T_{m}$, instead of $u_{\mathrm{rms}}$ and $T_{p}$. Case 2 tests started from an existing realistic scour hole distribution and each test represented a period during a 
storm with in- and then decreasing wave conditions. For case 2 the evolution of the scour depths with 'increased wave energy' differed from the scour depths with 'decreased wave energy'.

Besides the revision of the $K C$ number, the coefficients $A$ and $B$ were modified to improve the agreement of calculated and measured relative scour depths. The coefficients for scour only induced by waves (Sumer et al. 1992) are $A=0.03$ and $B=6$. The scour depths of case 1 fit well with the given coefficients of Sumer and Fredsøe (2001). However, the measured scour depths of case 2 fit better with calculated scour depths using coefficients $A=0.025$ and $B=7.5$. Thus, the initial seabed profile and the wave conditions (increased or decreased wave energy) play an important role in the scour hole formation.

Further PM experiments improved the knowledge of the time development of scour depending on the initial seabed conditions and the considered phase of the storm. These tests provide a basis for further developments and improvements of numerical models (Prepernau et al. 2008).

\subsection{Use of numerical model to overcome 2D physical model constraints}

The last case considers permeable, Low-Crested beach-parallel Structures (LCS) to obtain optimal information on wave heights, currents, water level and bottom evolution around the structure located on a sloping beach of $1 / 15$. The water depth at the front toe of the structure is $1 \mathrm{~m}$. Two types of structures were investigated: one submerged with a freeboard of $0.25 \mathrm{~m}$ and one emerged, with a crest height of $0.15 \mathrm{~m}$. The PM was tested in Barcelona's CIEM large scale flume with a mobile bed. A LCS with the same crosssection was modelled at the same scale with the morphodynamic model LIMORPH for waves, currents and beach morphodynamics. Nine irregular wave conditions generated with a Jonswap spectrum were tested for each structure, corresponding to three different wave heights and three wave steepnesses (Sierra et al. 2007; 2009).

First, the results of the two models were assessed and compared. The 2DV PM gave good results at the structural front because they reproduce shoaling, refraction, reflection and breaking processes. The PM results at its lee side are less good, because the PM does not simulate diffraction, longshore currents and sediment transport although it reproduces wave transmission, and it also does not accurately reproduce the water levels there, due to the piling-up effect of water, resulting in spurious results.

The NM did not give good results at the structural front because it does not simulate reflection although it reproduces shoaling, refraction and breaking. At its lee side, the NM allowed taking into account diffraction effects, which could not be included in the PM. Moreover, it gave more realistic set-up results at the leeside, because the NM did not feature the piling-up. However, the NM did not accurately represent the wave transmission. In terms of quality of the process representation for the LCS experiments, the PM and NM are complementary.

Although other possible approaches were analyzed, the actual $\mathrm{CM}$ approach consisted of selecting the areas where either PM or NM gave the better performance. The assessment of the areas of better performance was process-based, i.e. it took into account the physical processes that each model could simulate accurately at each area. In particular, PM results were selected in front of the structure and NM results at the leeside, expanding also the domain from 2DV to 2DH or Q3D.

This is a simple composite model as it combines information from a PM and a NM based on a priori knowledge of the processes in the models. A number of uncertainties exist even in this approach. For example there can be discontinuities between PM and NM bathymetries where they meet. Moreover it is 
difficult to calibrate the bottom evolution in the NM using the PM results as they follow different processes. It is also difficult to run both models simultaneously and to pass information between them as they run at different speeds. It was therefore impossible to run the models in an iterative way, as had been planned. This case study illustrates the difficulties in developing CM from a subjective, qualitative approach into a more objective, quantified approach.

\section{Reflections on Composite Modelling during CoMIBBS}

The case studies have indicated that both PM and NM can be used to model the processes and provide the essential quantified information on temporal and spatial evolution. Essential differences have also been addressed. For a PM the so-called similarity principle holds 'the same natural processes occur - essentially we just scale them' (Kamphuis 2000, van Os et al. 2004). One does not have to explicitly formulate the interactions - nature automatically reproduces them, given the drivers and constraints that the modeller provides. Therefore, a PM is particularly suited for studies involving non-linear interactions between water, structures and sediment. In these cases the physical model should be run at a large enough scale to minimise scale effects. In cases, such as with a detached breakwater, it is impossible to run at a scale large enough to have no scale effects and these must be accepted as a limitation of the PM. The case of one of the CoMIBBS partners, to analyse optimal scales of a PM, is therefore relevant.

In particular for situations where one process is considered, and the range of temporal and spatial scales of interest is limited, well-tested explicit mathematical equations are available, and have been implemented in NM codes, to mimic the dynamic processes of interest, given the 'drivers and constraints' (geometry, bathymetry, or forcing) that modellers provide. Characteristic for this is that the scales of interest are also the scales for which the NM is defined. For non-linear processes, the intrinsic interaction across scales when considering larger scales is not necessarily well-represented when explicit interaction descriptions are still hampered by insufficient understanding, one should then be careful to interpret the results for the larger scales. One should also be aware of this when using the current output from such shorter term models to drive transport models, if the interest is in larger spatial and temporal scales.

The above cases demonstrate that applying CM requires an explicit definition of the parameters that will be exchanged, including the quantification of the exchanged amount. This involves parameter, location and time, but also accuracy and representativity issues. Scales of variation and uncertainty are inherently present, and therefore their transfer from the one model to the other.

Aspects such as identifiability and measurability are associated with this. For example, the scalars water level, current components, wave height and wave period are well identifiable quantities, with large signal to noise ratio. The situation is more complex for concentrations of substances with a patchy character (e.g. suspended sediment) and if the interest is in larger time scales that the typical model time scales. Examples are spatial distribution of salinity and temperature, of sediment concentration, erosion and deposition patterns, bed height distribution, and their evolution in time. Careful assessment and quantification is important for successful application of CM.

The latter also is a key element in the development and application of analytical (regression) or behavioural models that directly link the response to the forcing, without trying to represent the processes involved (although knowledge of the processes is useful in guiding the choice of the parameters used in the fitting exercise). Coastal area NMs, however, solve the equations of fluid motion and calculate sediment 
concentrations, which are then advected and diffused, leading to erosion and deposition through conservation of mass.

Although each coastal morphological model is behavioural at some space and time scales, these models incorporate far more of the processes than the analytical models derived from fitting curves to physical model bathymetry data. It is therefore difficult to incorporate bathymetric results from a physical model of a small area into a coastal area numerical model of a large area.

The assessment of model behaviour and, in the end, model quality, requires explicit choices on which are the relevant quantities that need to be evaluated and compared, and how their differences (and which differences in which quantities we find acceptable) are quantified. This can be done in a quantified manner using a skill score. The Brier Skill Scores is practical and useful to immediately show whether resultants of a model simulation are or are not improved over those of a reference simulation. A variation on this is to use skill scores to choose an optimum PM or NM set-up that has a high skill score but does not require excessive resources to run.

Conservation of sediment is an important aspect in assessing the bathymetries from a PM and a NM. Nonconservation implies a sediment budget error, based on the relative difference between the changes in seabed elevation from the PM and the NM. Alternatively the difference between mean bed levels at the end of the PM and the NM runs can be used to quantify the level of agreement.

Unfortunately, there are still differences between the final bathymetries from the PM and the NM. The approach to deal with them may depend on the use to which the results will be put. A designer may choose to take a conservative approach and use the lower bathymetry at each point, which would lead to an apparent net sediment loss. Alternatively both bathymetries may be presented along with the changes between them, indicating the potential errors from using the NM versus the PM. If the PM was free from scale effects and measurement errors the changes would give a direct measure of the errors that come from using the NM.

These reflections emphasize a different aspect of the key element in composite modelling, namely the need for critical and quantitative assessment of the properties, the strengths and the weaknesses of the PM and NM that are being considered, plus the strict definition and quantification of the data that is used to couple the two when they are combined in composite modelling. This is an additional element of the Good Modelling Practice initiative developed about a decade ago.

\section{Good Modelling Practice}

Answering questions which involve geophysical processes almost invariably include modelling. The process from the initial question to the final answer and its interpretation in terms of fitness for use is often called the Modelling Cycle. In recent decades, the complexity of the Modelling Cycle has increased, for reasons that the models are more complex, and sometimes consist of chains of models, or because different persons play a role in this cycle. Scientists, engineers, computer specialists are often involved in the subsequent steps. The results of the analysis, forming the basis for the conclusions and answer to the originally posed question, travel the same way back. Along the road, data, perceptions and interpretations are communicated to guide the process. Understandably, miscommunication may easily occur.

The above issues were addressed explicitly in a project called Good Modelling Practice (GMP) (van Waveren et al. 1999). Its objective was to make the Modelling Cycle explicit through formulating questions, answers and interpretations. Often some standardised format is used, in order not to forget issues, and to 
promote re-use and referencing. The process and its decision points are then made transferable and referable to all involved. It can be easily discussed and adjusted, and the process can be backtracked. The EU project Harmoniqua extended this further by introducing computer-based tools to facilitate this process, given much attention to user friendliness, re-use and automated build-up of experiences and using this to improve the system (Refsgaard and Henriksen 2004, Old et al. 2005, Scholten et al. 2007, and the Harmoniqua project website at www.harmoniqua.org).

The systematic analysis and validation of the models that are used is a topic that is as directly related to the strengths and weaknesses of models and to the systematic process of model application outlined as Good Modelling Practice. An IAHR initiative to devise a hydraulic community approach to document the validity of the models is described in Dee et al. (1994). While the Validation initiative focuses on a critical analysis of the model, the GMP focuses on the whole model application train. They complement each other in enhancing the background and insight that is essential for successful modelling - and this is even more relevant for successful design of composite modelling.

\section{Deciding on Composite Modelling: a step-by-step process}

The above case studies have convinced the authors that a decision to apply CM is necessarily preceded by a problem analysis in the form of the question and answers, to estimate a priori the potential benefits of using $\mathrm{CM}$, or 'the integrated and balanced use of physical and numerical models'. The state-of-the-art of the tools for analysing the problem needs to be critically determined first. In different words the questions are: Which individual approaches and models are available? Which level of sophistications is required / which model or set of models? Are their strengths and weakness so that they can be satisfactorily applied to analyse the problem given the type of answer required?

The answers to these questions will or should give the information whether it makes sense to use $\mathrm{CM}$ : Is one type of model not adequate for analysing and solving the problems? Do physical and numerical models complement each other for this problem? Can different scales be separated in time and / or in space? Can the interfacing of the models and the exchange of data be defined uniquely, in terms of quantified parameters? Can an estimate be given of the uncertainty of the transferred data, so the transfer and effect of uncertainties from model to model can be estimated? (Oumeraci 1999, Kamphuis 2000 or van Os et al. 2004). The above provides the material to formulate a hypothesis on what we expect to gain by composite modelling for this problem, for example in terms of increased cost effectiveness, improved accuracy, reducing uncertainties, or tackling problems that could not reasonably be solved by a single model approach. The outcome of this process is an argued decision on whether or not CM is needed, or whether it is beneficial for modelling the problem at hand.

The advantage of approaching this conscientiously lies in the fact that we systematically build up the various elements into, for instance, an exchangeable and easily accessible library of physical and numerical models and their descriptions or metadata. This will provide updates on their capabilities, usefulness, and track records. It is part of Good Modelling Practice.

\section{Setting up a Composite Modelling experiment}

Consider that the previous steps have led to the conclusion that the case for $\mathrm{CM}$ is positive. To apply $\mathrm{CM}$ successfully, we again have to go through a careful decision process, which involves questions such as: 
What models do we use?, What is the overall domain in space and time for the problem?, What time and space scales will be covered by each model?, How to make external forcing consistent for PM and NM?, What data to transfer between the models, to provide forcing for the other model?, How these are best quantified?, What is the uncertainty in the data and how do these uncertainties influence the other model?, How do we quantify the results in terms of which quantities and in which norms?, How are PM and NM data evaluated against field data?, How can we visualise?, or How can a sensitivity analysis be conducted (this includes a data exchange protocol)?

A rigorous data exchange protocol is advised to assess the effect of the partial modelling elements on each other. Similarly, quantification and evaluation of the results is important to show the additional gain or benefit of applying composite modelling. By systematically addressing these issues and reporting the selections and arguments behind it the problem analysis process becomes transparent, and therefore transferable. This is not only important for the particular project itself. Sharing with colleagues internally and externally, and learning from each other will benefit from this as well.

\section{Conclusions}

The above case studies and their evaluation show that composite modelling is not straightforward but a complex research. It moreover is not the solution for all problems we cannot solve by either physical or numerical modelling. As a field, it still is science in its infancy. For problems that are suited for composite modelling, though, a well-designed approach can indeed extend the range of applications, or make the application cheaper, or more accurate. Composite modelling then is certainly worth the effort.

\section{Acknowledgements}

This work was supported by the European Community's Sixth Framework Programme through the grant to the budget of the Integrated Infrastructure Initiative HYDRALAB III, Contract no. 022441 (RII3). Co-funding was received from Deltares, HR Wallingford, Danish Council for Strategic Research (Project 09-066869), Bundesministerium für Umwelt, Naturschutz und Reaktorsicherheit (BMU-Germany) and Offshore-BürgerWindpark Butendiek GmbH \& Co. KG (Reference no. 0329973).

\section{Notation}
$A, B \quad$ current-dependent parameter
D diameter of pile or sphere
h water level
$H_{s} \quad$ significant wave height
$H_{b} \quad$ wave height at breaking
J position accuracy in Brier Skill Score
$k \quad$ turbulent kinetic energy
$K$ transport rate error in Brier Skill Score
KC Keulegan-Carpenter number 
L $\quad$ sediment budget error in Brier Skill Score

M normalisation term in Brier Skill Score

S scour depth

$S_{c} \quad$ current induced relative scour depth

$S_{c w} \quad$ wave and current induced scour depth

$T \quad$ representative period

$T_{m-1,0} \quad$ spectral average wave period

$T_{m} \quad$ mean wave period

$T_{p} \quad$ peak wave period

$u \quad$ representative velocity

$u_{\max } \quad$ maximum wave velocity

$u_{\text {rms }} \quad$ root-mean-square wave velocity

$x \quad$ downstream horizontal distance

$y \quad$ vertical elevation above initial seabed

$\alpha_{1} \quad$ width of low tide terrace

$\alpha_{2} \quad$ height of low tide terrace

\section{References}

Barthel, V., Funke, E.R. (1989). Hybrid modelling as applied to hydrodynamic research and testing. Recent Advances in Hydraulic Physical Modelling, 303-390, R. Martins, ed. NATO ASI Series E: Applied Sciences 165. Kluwer, Dordrecht NL.

Dee, D., Cunge, J., Labadie, G., Ruiz Mateo, A., Mathiesen, M., Price, R., Santos, M., Warren, R. (1994). Guidelines for documenting the validity of computational modelling software. IAHR, Delft NL.

DHI (2007). Litpack: Noncohesive sediment transport in currents and waves, User Guide. Danish Hydraulic Institute, Hørsholm DK.

Dixen, M. (2008). Interaction between seabed and scour protection. PhD. Thesis. Department of Mechanical Engineering, Coastal, Maritime and Structural Engineering Section. Technical University of Denmark, Lyngby DK.

Dixen, M., Sumer, B.M., Fredsøe, J. (2011). Numerical and experimental investigation of flow and scour around a half-buried sphere. Submitted for journal publication.

Fortes, C., Lemos, R., Neves, M.G., Reis, M.T., Santos, J.A., Pinheiro, L., Palha, A., Capitão, R., Sousa, I. (2009). Physical modelling of wave propagation and breaking in a flume using different geometric model scales. $2^{\text {nd }}$ Int. Conf. Application of Physical Modelling to Port and Coastal Protection (CoastLab08-Bari), 237-248, L. Damiani, M. Mossa, eds. IAHR, Madrid. 
Freire, P., Sancho, F., Oliveira, F.S.B.F. (2009). Composite modelling of sediment dynamics for propagating waves reaching coastal defences. $2^{\text {nd }}$ Int. Conf. Application of Physical Modelling to Port and Coastal Protection (CoastLab08-Bari), 125-135, L. Damiani, M. Mossa, eds. IAHR, Madrid.

Gerritsen, H., Sutherland, J., Deigaard, R., Sumer, B.M., Fortes, J, Sierra, J.-P., Prepernau, U. (2009). Guidelines for composite modelling of the interactions between beaches and structures. Final Report at http://www.hydralab.eu.

Gerritsen, H., Sutherland, J. (2011). Composite modelling. Hydralab: A users guide to hydraulic modelling and experimentation 6, 171-219, J. Kirkegaard, G. Wolters, J. Sutherland, R. Soulsby, L. Frostick, S. McLelland, T. Mercer, H. Gerritsen, eds. CRC Press/Balkema, Leiden NL.

Göthel, O. (2008). Numerical modelling of flow- and wave- induced scour around vertical circular piles. PhDThesis, Report-No. 76/2008. Institute of Fluid Dynamics, Leibniz University, Hannover.

Grunnet, N., Lohier, S., Deigaard, R. (2008a). Study of sediment bypass at coastal structures by composite modelling. Proc. $31^{\text {st }}$ Int. Conf. Coastal Engineering Hamburg, 1876-1887. World Scientific, Singapore.

Grunnet, N., Lohier, S., Deigaard, R., Brøker, I., Huiban, M. (2008b). Modelling of bypass of sediment at harbours. $9^{\text {th }}$ Int. Conf. Littoral 2008, Venice, 8p (CD-ROM).

Kamphuis, J.W. (2000). Designing with models. $27^{\text {th }}$ Int. Conf. Coastal Engineering, 19-32, B.L. Edge, ed. ASCE, Reston VA.

Lemos, R., Fortes, C.J.E.M., Gil, L., Neves, M.G. (2009). The influence of the geometric scale model on the physical modelling of the wave propagation and breaking in a flume. J. Coastal Res. SI 56, 583-592.

Lynett, P., Liu, P.L.-F. (2004). A two-layer approach to wave modelling. Proc. R. Soc. Lond. A 460, 26372669.

Old, G.H., Packman, J.C., Scholten, H. (2005). Supporting the European Water Framework Directive: The HarmoniQuA Modelling Support Tool (MoST). MODSIM 2005 Int. Congress Modelling and Simulation Melbourne, 2825-2831, A. Zerger, R.M. Argent, eds.

Obhrai, C., Sutherland, J. (2009). CoMIBBS physical model report. Report TR172, Release 2.0. HR Wallingford. Also HYDRALAB-III Report JRA1-08-06.

Oumeraci, H. (1999). Strengths and limitations of physical modelling in coastal engineering: Synergy effects with numerical modelling and field measurements. Hydralab: Workshop on Experimental Research and Synergy Effects with Mathematical Models, K.-U. Evers, J. Grüne, A. van Os, eds. Coastal Research Centre, Hannover.

Oumeraci, H. (2009). Composite modelling. CoMIBBS Report JRA1-09-10. Available at http://www.hydralab.eu.

Prepernau, U., Grüne, J., Schmidt-Koppenhagen, R., Wang, Z., Oumeraci, H. (2008). Large-scale model tests on scour around slender monopole under livebed conditions. $2^{\text {nd }}$ Int. Conf. Application of Physical Modelling to Port and Coastal Protection (CoastLab08) Bari, 215-224.

Refsgaard, J.C., Henriksen, H.J. (2004). Modelling guidelines - terminology and guiding principles. Advances in Water Res. 27(1), 71-82.

Reis, M.T., Neves, M.G., Fortes, C.J. (2008). Influence of physical model scale in the simulation of wave overtopping over a coastal structure. Proc. PIANC Mediterranean Days of Coastal and Port Engineering Palermo (CD-ROM). 
Scholten, H.A., Kassahun, J.C., Refsgaard, T., Kargas, C., Gavardinas, C., Beulens, A.J.M. (2007). A methodology to support multidisciplinary model-based water management. Environmental Modelling \& Software 22(5), 743-759.

Sierra, J.P., Gironella, X., Alsina, J.M., Oliveira, T.A.C., Cáceres, I., Mösso, C., Mestres, M. (2009). Physical and numerical modeling of beach response to permeable low-crested coastal structures. J. Coastal Res. SI 56, 1065-1069.

Sierra, J.P., Gironella, X., Sánchez-Arcilla, A., Sospedra, J., Alsina, J.M. (2007). Hybrid modelling of scouring-deposition in front of a coastal structure. J. Coastal Res. SI 50, 364-368.

Sumer, B.M. (2007). Mathematical modeling of scour: A review. J. Hydraulic Res. 45(6), 723-735.

Sumer, B.M., Fredsøe, J. (2001). Scour around pile in combined waves and current. J. Hydraulic Eng. 127(5), 403-411.

Sumer, B.M., Fredsøe, J., Christiansen, N. (1992). Scour around a vertical pile in waves. J. Wway., Port, Coastal and Ocean Eng. 118(1), 15-31.

Sutherland, J., Obhrai, C. (2009). CoMIBBS composite modelling report. Report TR173, HR Wallingford, also HYDRALAB-III Report JRA1-08-05.

Sutherland, J., Peet, A.H., Soulsby, R.L. (2004). Evaluating the performance of morphological models. Coastal Eng. 51(8-9): 917-939.

van den Boogaard, H., Gerritsen, H., Caires, S., van Gent, M. (2009a). Composite modelling by applying an inverse technique in analysing interactions between beaches and structures. $8^{\text {th }}$ Int. Conf. Hydroinformatics, Concepción, Chile, paper 188a178, 10 pages (CD-ROM).

van den Boogaard, H., Gerritsen, H., Caires, S., van Gent, M. (2009b). Wave attack on sea defences: Potential benefits of a composite modelling approach. $33^{\text {rd }}$ IAHR Congress Vancouver (10443), 1006-1013.

van Os, A.G., Soulsby, R.S., Kirkegaard, J. (2004). The future role of experimental methods in European hydraulic research: towards a balanced methodology. J. Hydraulic Res. 42(4), 341-356.

van Waveren, R.H., Groot, S., Scholten, H., van Geer, F.C., Wösten, J.H.M., Koeze, R.D., Noort, J.J. (1999). Good modelling practice handbook. STOWA Report 99-05. Dutch Dept. of Public Works, Inst. for Inland Water Management and Waste Water Treatment, Report 99.036. 